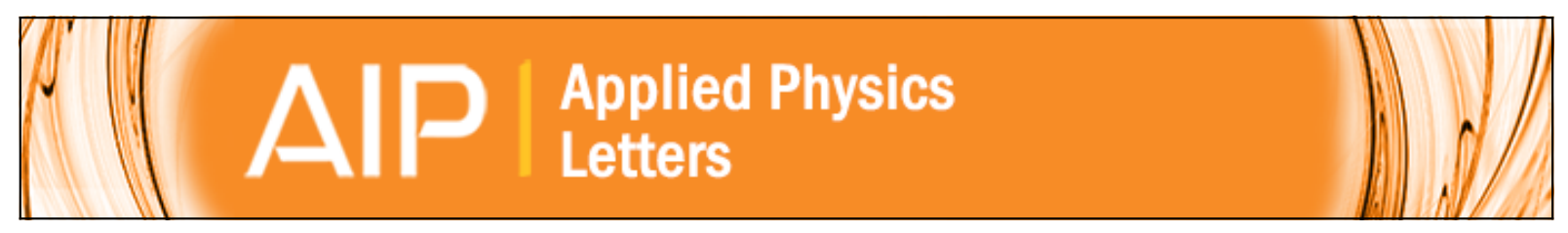

\title{
Sound-resonance hydrogen sensor
}

Shuxiang Dong, Feiming Bai, JieFang Li, and Dwight Viehland

Citation: Applied Physics Letters 82, 4590 (2003); doi: 10.1063/1.1586994

View online: http://dx.doi.org/10.1063/1.1586994

View Table of Contents: http://scitation.aip.org/content/aip/journal/apl/82/25?ver=pdfcov

Published by the AIP Publishing

\section{Over 700 papers \& presentations on multiphysics simulation}

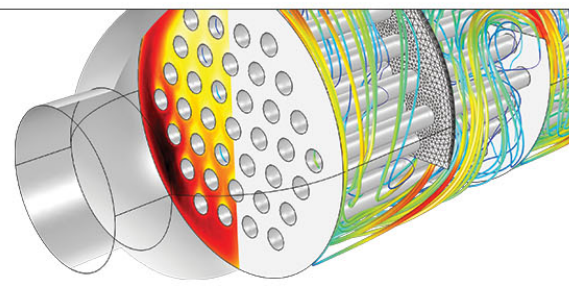




\title{
Sound-resonance hydrogen sensor
}

\author{
Shuxiang Dong, ${ }^{\text {a) }}$ Feiming Bai, JieFang Li, and Dwight Viehland \\ Materials Science and Engineering, Virginia Tech, Blacksburg, Virginia 24061
}

(Received 28 February 2003; accepted 28 April 2003)

\begin{abstract}
A hydrogen sensor is reported in which a small piezoelectric-sound-resonance-cavity (PSRC) is used as the sensing element. Detection utilizes sound resonance and acoustic property differences between $\mathrm{H}_{2}$ and air as a sensing mechanism. Changes in $\mathrm{H}_{2}$ concentration result in a shift of the sound-resonance state of the PSRC. Preliminary experiments have demonstrated a sensitivity limit of $8 \mathrm{ppm}$, a fast response time $\sim 1.5$ second, and detection capabilities over a broad concentration range $10^{-5}<n<0.2$. (C) 2003 American Institute of Physics. [DOI: 10.1063/1.1586994]
\end{abstract}

Gas sensors have been developed for a wide range of air-borne hazardous species, including flammable and toxic gases. Current solid-state gas sensors can be classified into three types based upon their sensing mechanism, ${ }^{1-6}$ including: (i) Semiconducting ceramics that use electronic conductivity (i.e., surface resistivity), (ii) solid electrolytes that use ionic conductivity, and (iii) solid-state dielectrics that use capacitance. The response times of these solid-state gas sensors are quite slow, dependent upon the equilibrium adsorption of gases onto solid surfaces. They often can detect the presence of a gas; however, they have compromised signal sensitivity to gas concentrations and reduced response times. They are not useful for rapid quantitative detection over a significant concentration range.

Also, solid-state ceramic gas sensors often require relatively high operational temperatures $\left(150\right.$ to $\left.500{ }^{\circ} \mathrm{C}\right)$, making them unsuitable for the detection of flammable gases and liquids. As an alternative, polymer-based gas sensors have been developed which use an admittance (capacitance and conductance) detection method. ${ }^{7,8}$ These types of solid-state gas sensors can be operated at room temperature. However, they have very slow response times, as the admittance changes are dependent upon adsorption of gas species onto polymer surfaces. Again, they lack rapid, quantitative detection capabilities.

There are other alternative solid-state sensors based upon (i) optical/photoacoustic properties, (ii) surface acoustic waves, (iii) palladium mesowire, (iv) piezoelectric, and (v) microelectromechanical systems (semiconducting) mechanisms. The main problems associated with these types of sensors are a $\mathrm{H}_{2}$ saturation problem, and/or a very low operational temperature (i.e., photoacoustic gas sensor). ${ }^{9-17}$

Clearly, other types of gas sensors are needed which have enhanced sensitivity, quicker response times, and quantitative detection capabilities over a wider concentration range for $\mathrm{H}_{2}$ leak detection, such as health monitoring of aerospace fuel tanks, in which $\mathrm{H}_{2}$ is a known detected gas. The acoustic properties of gases have been used as a sensing mechanism for air pollutions ${ }^{18}$ and humidity detection (using surface acoustic wave). ${ }^{19}$ We will show that it is also a good sensing mechanism for $\mathrm{H}_{2}$ detection, as there are significant

${ }^{a)}$ Electronic mail: sdong@vt.edu differences in the sound speeds, mass densities, and acoustic impedances between $\mathrm{H}_{2}$ and air.

Figure 1 illustrates the concept of a PSRC sensor. Two disk-type piezoelectric thin layers are placed at each end of a small cavity (cylindrical type), in which one piezoelectric film has a center hole. One of the piezoelectric films is used to produce a bending vibration under a small applied ac voltage signal $\left(V_{\text {ac }}\right)$, forcing the air in the cavity to vibrate. At resonance, the air will be in a standing wave sound resonance state. The second piezoelectric element at the opposite end of the cavity acts as a sensing element, capable of monitoring the changes of both the acoustic intensity $(\Delta I)$ and frequency of the standing wave $(\Delta f)$. The piezoelectric sensor will produce a voltage signal $\left(V_{s}\right)$ and phase signal $\left(P_{s}\right)$ which are proportional to $\Delta I$ and $\Delta f$.

The resonance frequency of a cavity containing air of a nominal composition is

$$
f_{0}=K C_{\text {air }},
$$

where $K$ is a geometric parameter related to the structure and size of the cavity, and $C_{\text {air }}$ is air sound velocity. When $\mathrm{H}_{2}$ enters the cavity, supposing the $\mathrm{H}_{2}$ concentration percentage to be $n$ ( $n$ is a small quantity), and the air concentration percentage to be $(1-n)$, the average density of the air and hydrogen gas can be estimated as $\bar{\rho}=n \rho_{\mathrm{H}_{2}}+(1-n) \rho_{\text {air }}$. According to ideal gas laws, the average sound velocity of the air and hydrogen gas is $\bar{C} \approx C_{\text {air }} / \sqrt{1-n}$ (when $n \ll 1$ ). As a

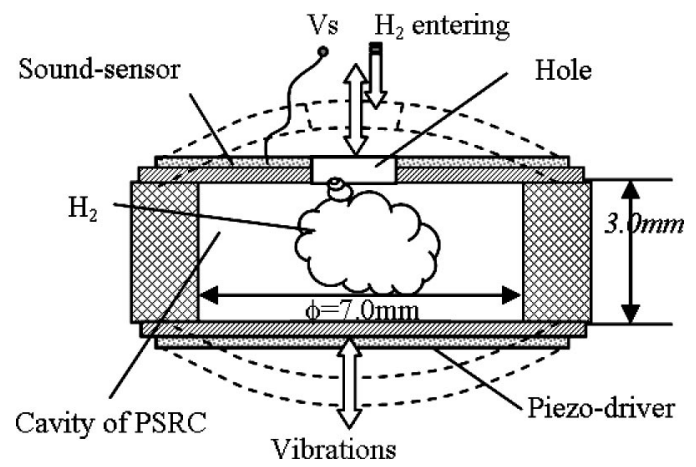

FIG. 1. Illustration of the concept of the PSRC sensor. The bottom piezodriver produces a standing wave sound-resonance state in the cavity of PSRC. The top sound-sensor senses the changes of both acoustic intensity $(I)$ and frequency $\left(f_{o}\right)$ of the standing wave caused by $\mathrm{H}_{2}$ gas. 
result, the resonance frequency shift and acoustic characteristic impedance change of the cavity caused by $\mathrm{H}_{2}$ are

$$
\begin{aligned}
& \Delta f_{0}=K\left(\bar{C}-C_{\text {air }}\right) \approx \frac{n}{2-3 n / 2} K C_{\text {air }} \\
& \Delta(\rho C)=\bar{\rho} \bar{C}-\rho_{\text {air }} C_{\text {air }} \approx n\left[(1+n / 2) \rho_{\mathrm{H}_{2}}-\rho_{\text {air }} / 2\right] C_{\text {air }} .
\end{aligned}
$$

The resonance frequency shift $\Delta f_{0}$ and acoustic characteristic impedance change $\Delta(\rho C)$ are thus directly proportional to the $\mathrm{H}_{2}$ concentration $n$ when $\mathrm{H}_{2}$ concentration $n$ is a small quantity.

Suppose that the piezoelectric driving element is operated at a constant vibration velocity, which causes the gas particles to vibrate at a constant average speed $v_{e}$ in the cavity. As a result, any change in the acoustic characteristic impedance, $\Delta(\rho C)$, will cause a change in acoustic intensity radiation $(\Delta I)$ :

$$
\Delta I=\Delta(\rho C) v_{e}^{2} .
$$

[Note, $\Delta(\rho C)$ is a negative value because $\rho_{\mathrm{H}_{2}} \ll \rho_{\text {air }}$.] According to Eq. (4), a cavity containing a gas of low acoustic impedance will result in a lower acoustic intensity radiation if the gas particles vibrate at a constant average speed $v_{e}$. Because the voltage signal $V_{s}$ induced from the sensing element is $\sim \Delta I$ and $\Delta I \sim n$ [by Eqs. (3) and (4)], a small change in $\mathrm{H}_{2}$ concentration $n$ will produce a near linearly proportional change in $V_{s}$. Consequently, there exists a direct linear signal between the acoustic properties of the cavity and the $\mathrm{H}_{2}$ concentration there within, which can be measured by the voltage output from a piezoelectric sensing element. The air acoustic speed and mass density are $330 \mathrm{~m} / \mathrm{s}$ and $1.29 \mathrm{~kg} / \mathrm{m}^{3}$, whereas these of $\mathrm{H}_{2}$ are $1280 \mathrm{~m} / \mathrm{s}$ and 0.089 $\mathrm{kg} / \mathrm{m}^{3}$, respectively. This big difference in acoustic properties offers significant potential to detect changes in $\mathrm{H}_{2}$ concentration with high sensitivity.

A PSRC prototype gas sensor with an operational frequency of $4.7 \mathrm{kHz}$ was built, as shown in Fig. 1. This prototype consisted of two PZT thin layers with a diameter of 8 $\mathrm{mm}$ and a thickness of 100 microns, and a plastic cavity of 7 $\mathrm{mm}$ in diameter and $3 \mathrm{~mm}$ in height. An effective method to operate a PSRC sensor is at $90^{\circ}$ or $-90^{\circ}$ phase state under constant frequency conditions. When $\mathrm{H}_{2}$ enters the cavity, a phase shift, $\Delta P_{s}$, of the resonance state will occur. $\mathrm{H}_{2}$ also will result in an acoustic impedance change, which can be detected as a voltage change $\Delta V_{s}$.

The response of the PSRC sensor to changes in $\mathrm{H}_{2}$ concentration was determined in a $\mathrm{H}_{2}$ test chamber. A small ac $(4.794 \mathrm{kHz})$ voltage signal of $0.1 V_{\text {rms }}$ was used to drive a piezoelectric element at one end of the cavity. A lock-in amplifier was used to monitor the signal from the sensing element of the PSRC sensor. The temperature and relative humidity were held constant in the test chamber. To characterize the PSRC sensors capability of quantitative detection, the $\mathrm{H}_{2}$ concentration in test chamber was varied over a wide concentration range from $10^{-6}<n<0.2$.

Figures 2(a) and 2(b) show the sensor output phase angle and voltage for the PSRC prototype as a function of $\mathrm{H}_{2}$ concentration. The $\mathrm{H}_{2}$ concentration growth rate was held constant at $\Delta n / \Delta t=0.091(\mathrm{ppm} / \mathrm{sec})$. In Figs. 2(a) and 2(b),

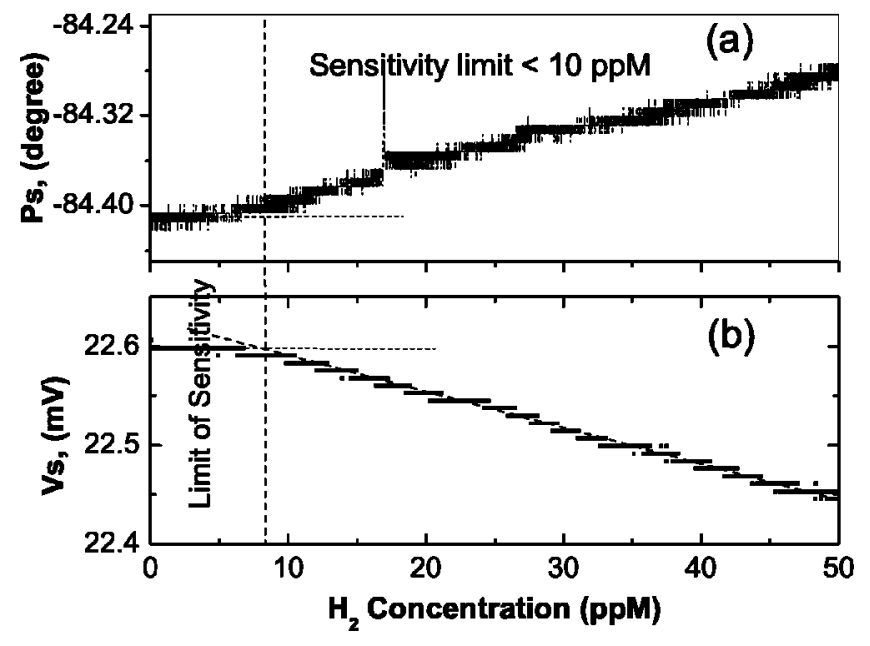

FIG. 2. PSRC sensor (a) phase and (b) voltage as a function of $\mathrm{H}_{2}$ concentration at low concentration of $\mathrm{H}_{2} \quad\left(\mathrm{H}_{2}\right.$ generation by electrochemical method). Constant circumstance temperature and humidity: $16{ }^{\circ} \mathrm{C}$ and $36 \%$, respectively.

it can be seen that both the voltage and phase angle can detect changes in $\mathrm{H}_{2}$ concentration of less than $10 \mathrm{ppm}(\sim 8$ $\mathrm{ppm})$. Above this sensitivity limit, the voltage and phase output were both found to be linear functions of $\mathrm{H}_{2}$ concentration. For example, an $\mathrm{H}_{2}$ concentration change of $42 \mathrm{ppm}$ resulted in a phase shift of $0.113^{\circ}$ and a voltage change of $-0.16 \mathrm{mV}$. These correspond to relative changes of $\Delta P_{s} / P_{s 0}=1.34 \times 10^{-3}$ and $\Delta V_{s} / V_{s 0}=-7.1 \times 10^{-3}$, where $P_{o}=-84.412$ and $V_{s 0}=22.6 \mathrm{mV}$.

The performance of the prototype PSRC sensor was evaluated over wider and higher $\mathrm{H}_{2}$ concentration ranges: (i) $0-4000 \mathrm{ppm}$ and (ii) $0 \%-10 \%$. Figures 3(a) and 3(b) show the voltage and phase angle as a function of time over an intermediate $\mathrm{H}_{2}$ concentration range. The concentration was increased by $670 \mathrm{ppm} / \mathrm{step}$, using a total of six steps and a total $\mathrm{H}_{2}$ concentration change of $4025 \mathrm{ppm}$. This resulted in a maximum total phase shift of $2.07^{\circ}$ and a voltage change of $-0.206 \mathrm{mV}$. The corresponding relative changes are

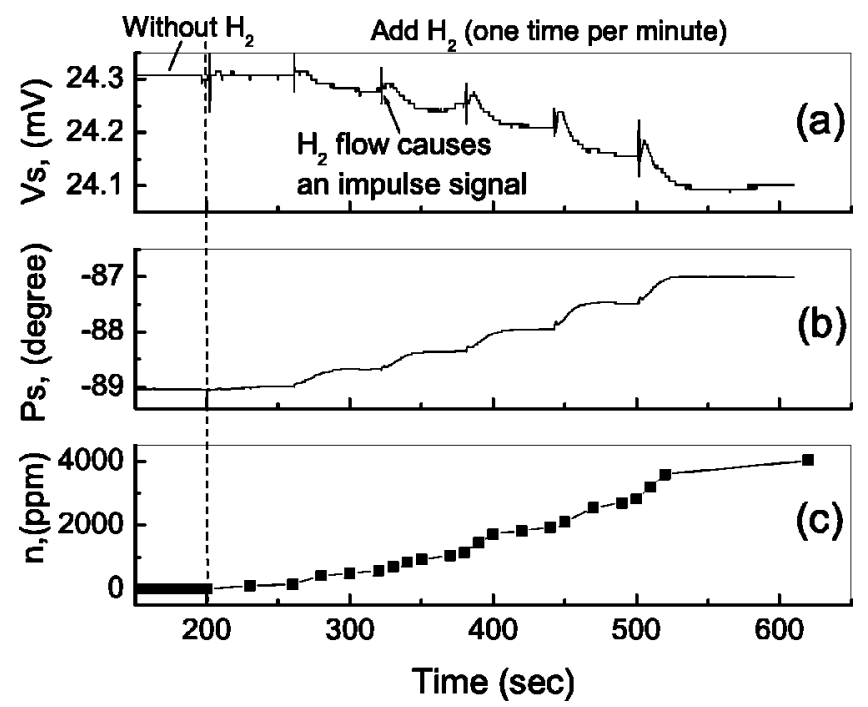

FIG. 3. Measurements in the $\mathrm{H}_{2}$ concentration range of 0 to $4000 \mathrm{ppm}$ : (a) voltage and (b) phase as a function of time for the PSRC sensor, (c) voltage as a function of time for the conventional ceramic gas sensor (Riken Keiki, Model SD.70). Constant circumstance temperature and humidity: $16^{\circ} \mathrm{C}$ and $31 \%$, respectively. 


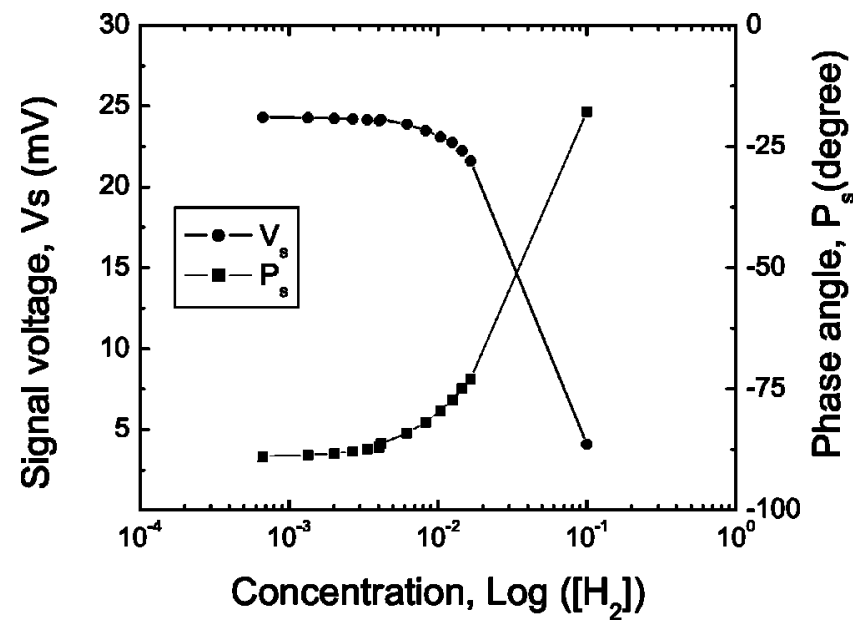

FIG. 4. Voltage and phase as a function of $\mathrm{H}_{2}$ concentration for the PSRC sensor in the concentration range of $6 \times 10^{-4}<n<0.2$. Constant circumstance temperature and humidity: $16^{\circ} \mathrm{C}$ and $32 \%$, respectively.

$\Delta P_{s} / P_{s 0}=2.32 \times 10^{-2}$ and $\Delta V_{s} / V_{s 0}=-8.48 \times 10^{-3}$, where $P_{s 0}=-89.06^{\circ}$ and $V_{s 0}=24.30 \mathrm{mV}$. The various impulse signals in Fig. 3(a) are due to the step-wise manner in which the $\mathrm{H}_{2}$ concentration was increased. Figure 3(c) shows the $\mathrm{H}_{2}$ concentration as a function of time, determined using a conventional semiconducting ceramic sensor. Even though $\mathrm{H}_{2}$ was introduced into the test chamber in a step-wise manner, its time-dependent response was smooth. This reflects the fact that its response time is significantly longer than the time differential between steps in the $\mathrm{H}_{2}$ concentration.

Figure 4 shows the voltage and phase angle as a function of $\mathrm{H}_{2}$ concentration. The voltage and phase angle can be seen to be proportional to the $\mathrm{H}_{2}$ concentration over a wide concentration range of $6 \times 10^{-4}<n<0.1$. This resulted in a maximum total phase shift of $\sim 75^{\circ}$ and a voltage change of $\sim-20 \mathrm{mV}$. (As a comparison, the semiconductor ceramic sensor became saturated and its voltage independent of $\mathrm{H}_{2}$ concentration for $n \geqslant 8.3 \times 10^{-3}$ ). Theoretically, the gas in the cavity may be $\mathrm{H}_{2}$ of $100 \%$. The response time of the PSRC sensor to $\mathrm{H}_{2}$ concentration changes was very rapid; typically, it is about $1.5 \mathrm{~s}$ (see Fig. 5). Note, the cavity of PSRC sensor should be kept at a constant temperature and humidity condition, as it is also sensitive to these variations.

In conclusion, we have demonstrated a PSRC concept in $\mathrm{H}_{2}$ sensing which uses sound resonance and acoustic property differences of gases as a sensing mechanism. It is a compact, robust, and light weight sensor. It has very low working voltages $(<100 \mathrm{mV})$ and extremely low-power consumptions $(<100 \mu \mathrm{W})$. Investigations of a PSRC prototype have demonstrated a sensitivity limit of $8 \mathrm{ppm}$, a fast response time $\sim 1.5$ second, and a signal (phase and voltage) that is a linear function of $\mathrm{H}_{2}$ concentration in the low con-

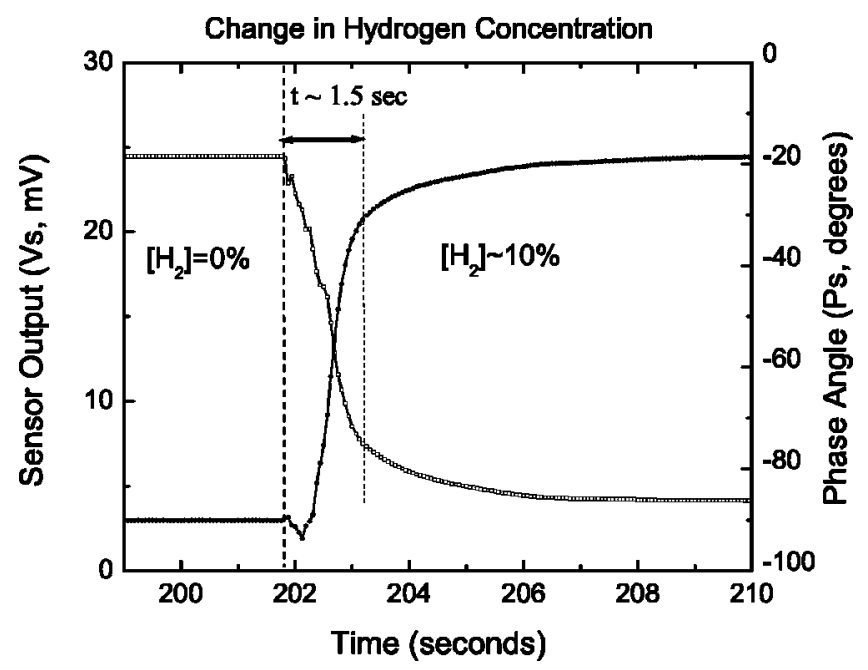

FIG. 5. Illustration of response time of PSRC sensor to a change in $\mathrm{H}_{2}$ concentration.

centration range, and that is also proportional to $\mathrm{H}_{2}$ concentration over a wide range of $\mathrm{H}_{2}$ concentrations between 8 $\times 10^{-6}<n<0.2$.

This research was supported by the NASA, Marshall Space Flight Center. Any opinions, findings, and conclusions or recommendations expressed in this material are those of the author(s) and do not necessarily reflect the views of NASA.

${ }^{1}$ D. D. Lee and D. S. Lee, IEEE Sensors J. 1, 3 (2001).

${ }^{2}$ K. Ihokura and J. Watson, The Stannic Oxide Gas Sensors (CRC Press, Boca Raton, 1994).

${ }^{3}$ D. D. Lee, Chem. Sens. Technol. 5, 79 (1994).

${ }^{4}$ C. Xu, J. Tamaki, and N. Yamazoe, J. Mater. Sci. 27, 963 (1992).

${ }^{5} \mathrm{~W}$. Weppner, Proceedings of the Second International Meeting on Chemical Sensors, Bordeaux, France, 1986, p. 59.

${ }^{6}$ A. D. John, Analytical Chemistry Handbook (McGraw-Hill, New York, 1995).

${ }^{7}$ M. J. C. Petty and R. Casalini, Eng. Sci. Edu. J. 99, (2001).

${ }^{8}$ R. Casalini, J. Nagel, U. Oertel, and M. C. Petty, J. Phys. D 31, 3146 (1998).

${ }^{9}$ S. L. Firebaugh, K. F. Jensen, and M. A. Schmidt, J. Microelectromech. Syst. 10, 232 (2001).

${ }^{10}$ M. A. Butler, Appl. Phys. Lett. 45, 1007 (1984).

${ }^{11}$ I. D. Avramov, S. Kurosawa, M. Rapp, P. Krawczak, and E. I. Radeva, IEEE Trans. Microwave Theory Tech. 49, 827 (2001).

${ }^{12}$ I. A. Ges and B. A. Budkevich, Proceedings of the 1999 Joint Meeting EFTF-IEEE IFCS, 1999, p. 1070.

${ }^{13}$ F. Favier, E. C. Walter, M. P. Zach, T. Benter, and R. M. Penner, Science 21, 2227 (2001).

${ }^{14}$ H. I. Chen, Y. I. Chou, and C. Y. Chu, Sens. Actuators B 85, 10 (2002).

${ }^{15}$ S. V. Slobodchikov, D. N. Goryachev, K. M. Salikhov, and O. M. Sreseli, Semiconductors 33, 339 (1999).

${ }^{16}$ W. P. Kang and Y. Gurbuz, J. Appl. Phys. 75, 8175 (1994).

${ }^{17}$ W. C. Liu, H. J. Pan, H. I. Chen, K. W. Lin, S. Y. Cheng, and K. H. Yu, IEEE Trans. Electron Devices 48, 1938 (2001).

${ }^{18}$ R. T. Muehleisen, ARLO 3, 118 (2002).

${ }^{19}$ E. R. Braga, A. Y. Nakano, and M. P. Cunha, Proceedings of the S BMO/ IEEE M TT-S IMOC'99, 1999, p. 342. 